\title{
Konturen eines Plattform-Urbanismus
}

\author{
Soziale und räumliche Ausprägungen eines digital divide am Beispiel Smart Mobility
}

\author{
Sybille Bauriedl \\ Henk Wiechers
}

Smart Cities sind mit großen Versprechen hinsichtlich einer verbesserten urbanen Lebensqualität durch digitale Technologien verbunden. Diese technologieoptimistische Stadtentwicklung schließt an Modernisierungsversprechen der „unternehmerischen Stadt" an. Vielfältige Versorgungsaufgaben in Städten werden mittlerweile über Onlineplattformen vermittelt, die von internationalen IT-Konzernen betrieben und durch digitale Infrastrukturen im öffentlichen Raum unterstützt werden. Dieser Prozess wird in der Stadtforschung als Plattform-Urbanismus bezeichnet.

Der Beitrag geht von der These aus, dass Smart-City-Infrastrukturen und -Dienstleistungen räumlich exklusiv realisiert werden. Er stellt am Beispiel von Smart Mobility in europäischen Städten die vielfältigen plattformvermittelten Mobilitätsangebote vor und diskutiert, welche Nachfrage sie ansprechen und ob damit urbane Mobilitätsdefizite und sozialräumliche Segregation behoben werden können oder aber durch einen ungleichen Digitalisierungszugang Mobilitätsungerechtigkeit verstärkt wird. Diese Frage wird mit Referenz auf Studien zu Geschlechterverhältnissen im Mobilitätsalltag diskutiert und dient als Grundlage für unsere Abschlussthesen, die die Folgen von Standardisierung, Normierung und Universalisierung als Merkmale des Plattform-Urbanismus in Europa kritisch beleuchten.

Ersteinreichung: 21. Mai 2020; Veröffentlichung online: 23. April 2021

An English abstract can be found at the end of the document.

\section{Einleitung}

Die kritische Stadtforschung hat in den vergangenen Jahren die vielfältigen Ausformungen und Ambivalenzen einer digitalisierten Stadtentwicklung aufgespürt und Smart-City-Strategien als Ausdruck unternehmerischer Stadtpolitik beschrieben (vgl. Söderström et al. 2014; Bauriedl/Strüver 2018). IT-Konzerne wie IBM, Cisco und Telekom sowie Plattformkonzerne wie Alphabet, Facebook, Amazon, Airbnb und Uber haben Städte als neue Märkte entdeckt, und insbesondere wettbewerbsorientierte Stadtregierungen, die sich an den Idealen einer „unternehmerischen Stadt“ orientieren (vgl. Heeg/ Rosol 2007), sehen deren Smart-City-Produkte und -Dienstleistungen zunehmend als Säulen ihrer Stadtentwicklungsstrategie. Damit hat ein sehr dynamischer Prozess der Integration digitaler Technologien und Infrastrukturen im öffentlichen und privaten Raum eingesetzt, der in der Stadtforschung als „smart urbanism“ (Marvin et al. 2016) und „platform urbanism“ (Barns 2020) beschrieben wird. An dieser Stelle wollen wir die Beobachtung 
eines Plattform-Urbanismus als neue Qualität digitaler Transformation aus der Perspektive der kritischen Stadtforschung kenntlich machen.

In diesem Beitrag beschäftigen wir uns mit der jüngsten Stadtforschungsdebatte zum Plattform-Urbanismus und legen dabei einen sektoralen Fokus auf urbane Mobilität[1] und einen sozialen Fokus auf Geschlechterverhältnisse. Sektorale Plattformen und plattformvermittelte Dienstleistungen sind ein nicht mehr neues, aber immer relevanteres Phänomen der Organisation von Alltagsaktivitäten im Stadtraum. Mobilitätsplattformen sind aktuell besonders sichtbar durch ihre Infrastrukturen (reservierte Parkplätze, Fahrzeugflotten) und ihre Alltagsnutzung sowie aufgrund der öffentlichen Debatte zu den positiven Potenzialen einer Smart Mobility, die von vielen Stadtregierungen und Mobilitätsunternehmen vorangetrieben wird. Smart Mobility (und damit verbundene Multimodalitäten) wird mittlerweile innerhalb der Mobilitätsforschung und Verkehrspolitik als hegemoniale Alternative zur Automobilität verhandelt.

Die daraus resultierenden räumlichen, sozialen und politischen Dynamiken machen wir nachvollziehbar, indem wir einerseits darstellen, welche neuen Mobilitätsangebote über Plattformen bereitgestellt werden, und andererseits zeigen, welche Mobilitätsnachfrage damit generiert beziehungsweise ignoriert wird. Angesichts der Beobachtung, dass (urbane) Mobilität sozial und räumlich ungleich verfügbar ist (Law 1999), haben wir aus vorliegenden Studien Erkenntnisse darüber zusammengeführt, ob neue Smart-Mobility-Dienstleistungen alle Verkehrsteilnehmer_innen ansprechen oder bereits privilegierte Gruppen und Stadträume priorisieren und damit städtische Ungleichheiten reproduzieren. In diesem Beitrag führen wir auf Basis einer Literaturarbeit die aktuellen Positionen der kritischen Stadtforschung zu Plattform-Urbanismus, der Mobilitätsforschung zu Smart Mobility Governance und der Feministischen Geographie zu vergeschlechtlichter Mobilität zusammen und arbeiten damit die Narrative und Materialisierungen des Plattform-Urbanismus am Beispiel von Smart Mobility heraus.[2] Wir zeigen auf diese Weise, dass die positiv konnotierten Fortschritts- und Modernisierungsversprechen von Smart Mobility nur zum Preis von Normierung, Standardisierung und Universalisierung spezifischer Vorstellungen von Urbanität zu haben sind.

Unsere Schlussfolgerungen konzentrieren sich auf europäische Großstädte, da erstens plattformvermittelte Mobilitätsdienstleistungen hier eine sehr große Verbreitung haben und sich Automobilkonzerne auf Großstädte als neuen Markt für Smart Mobility konzentrieren, zweitens die Nutzung des öffentlichen Nahverkehrs und nicht-motorisierter Verkehrsmittel im Vergleich zum Mobilitätsalltag in anderen Regionen einen höheren Stellenwert hat und drittens die Regulationsmechanismen einer urbanen Mobilitätstransformation stärker ausgeprägt sind (Docherty et al. 2018). Europäische Städte sind durch vielfältige Formen räumlicher Segregation und sozialer Polarisierung geprägt, die mit digitalen Infrastrukturen und plattformvermittelten Mobilitätsangeboten nicht automatisch behoben werden (vgl. Graham 2002). Es besteht hingegen das Risiko, dass eine digitale Transformation in Städten soziale Ungleichheit und sozialräumliche Ungerechtigkeit sogar verschärft (vgl. WBGU 2019: 1).

Diesen Potenzialen und Risiken des Plattform-Urbanismus gehen wir in fünf Abschnitten nach. Darin skizzieren wir zuerst den Stellenwert der 
Plattformökonomie für Urbanisierungsprozesse und damit verbundene sozialräumliche Differenzstrukturen (digital divide). Danach stellen wir die aktuelle Angebotsstruktur und die dominierenden Akteure von plattformvermittelten Mobilitätsdienstleistungen in europäischen Großstädten vor, um im nächsten Schritt die damit verbundenen unterschiedlichen Zugänglichkeiten zu Mobilitätsoptionen zu diskutieren (multimodal divide). Diese Argumente vertiefen wir mit Blick auf die Differenzproduktion und Identitätsnormierung von Geschlechterverhältnissen (gendered multimodal divide) mit Bezug auf die feministische Mobilitäts- und Stadtforschung. Die Konzepte von digital divide, multimodal divide und gendered multimodal divide bauen aufeinander auf. Gendered multimodal divide beschreibt schließlich die entlang vergeschlechtlichter Differenzlinien ungleich verteilten Zugangsmöglichkeiten und Ausstattungen im Mobilitätssektor. Wir sprechen hier explizit nicht von geschlechtsspezifischer (binär gedacht weiblicher oder männlicher) Mobilität, sondern von vergeschlechtlichter Mobilität, die entlang von geschlechtlicher Arbeitsteilung, Rollenzuweisungen und Geschlechtsidentität organisiert ist (Hanson 2010; Singh 2019). In unseren Schlussfolgerungen fassen wir die sozialräumlichen Implikationen der genannten Konzepte und der Plattformisierung urbaner Mobilität zusammen und stellen alternative, ermöglichende Potenziale einer nachhaltigeren Stadtentwicklung zur Diskussion.

\section{Plattform-Urbanismus als Ausdruck einer technokapitalistischen Modernisierung von Städten}

Die Argumentation dieses Beitrags beruht auf unserer laufenden Studie zu Plattform-Urbanismus,[3] die von der These geleitet ist, dass Smart-CityInfrastrukturen und -Dienstleistungen räumlich exklusiv realisiert werden. Das zeigt sich darin, dass digitale Infrastrukturen im Rahmen des SmartCity-Wettbewerbs europäischer Städte eher der Aufmerksamkeitsproduktion für innovative digitale Infrastrukturen in ausgewählten Stadtteilen als der Versorgung der Grundbedürfnisse der gesamten Stadtbevölkerung dienen. Digitale Plattformen und Smart Mobility verstehen wir in diesem Sinne als hochpolitische Innovationen, da sie zentrale Aspekte der sozialen Gleichheit, Nachhaltigkeit und Demokratie, des Rechts auf Stadt und der Gestalt des öffentlichen Raums maßgeblich beeinflussen.

Digitale Plattformen werden im urbanen Alltag vielfältig genutzt und dringenin immermehr private und öffentliche Lebensbereichevor. Plattformenvermitteln Informationen (z. B. Stadtpläne, Wetterprognosen), Arbeitsleistungen (z. B. Lieferdienste, Fahrdienste) und temporäre Nutzungsrechte (z. B. für Fahrzeuge) und Güter (z. B. Gebrauchtwarenversteigerung). Informationen, Arbeit oder Nutzungsrechte werden über Plattformen zu Tauschwaren, die gegen Geld und/oder persönliche Daten und/oder Werbung gehandelt werden. Durch die Plattformvermittlung werden diese Tauschwaren der Einbettung in zwischenmenschliche Vertrauensverhältnisse, der Interaktionen und in weiten Teilen auch der zivilgesellschaftlichen Kontrolle entzogen, und gleichzeitig generieren sie neue Verbindungen und Interaktionen. Plattformen sind dennoch mehr als Vermittler zwischen Angebot und Nachfrage. Auch wenn Unternehmen wie Uber oder Airbnb sich als reine Technologieunternehmen präsentieren, die Plattformen bereitstellen, sind sie auch Transport- und 
Immobilienunternehmen und nehmen über ihre Algorithmen Einfluss auf Konsument_inneninteressen, Arbeitsverhältnisse und Hotspots ihrer Dienstleistungen in den Städten, in denen sie ihre Dienste anbieten (dürfen). Die Algorithmenprogrammierung nimmt eine Selektion darüber vor, was Kund_innen zu sehen bekommen, und lässt das dahinterliegende Geschäftsmodell nur schwer erkennen.

Die auf den Plattformen angebotene Auswahl kann fix vorprogrammiert, individuell zugeschnitten oder durch Künstliche Intelligenz generiert werden (wenn viele Kund_innen nach A das Angebot B wählen, wird nach der nächsten Wahl von A ebenfalls B angeboten werden; Angebote können je nach Standort variieren etc.). Dahinter liegen also einerseits gezielte Strategien der Plattformbetreiber, andererseits vorurteilsverstärkende Algorithmen, die auf Big Data beruhen.

Digitale Plattformen sind für den Lebens- und Arbeitsalltag wie auch für dieStadtforschung ein relativ neues Phänomen, das aus diversen Perspektiven relevant ist. Plattformen stellen nicht nur eine innovative Technologie dar und ein neues Geschäftsmodell, sie funktionieren auch als virtuelle Kontaktzone, in der Entscheidungen getroffen werden; sie bilden eine soziotechnische Infrastruktur, die Normierungen vornimmt, sie dienen als Disziplinierungsinstrument für prekäre Arbeitsverhältnisse der Dienstleistungsanbieter, sie werden von den Betreibern neben der nachgefragten Dienstleistung auch zur Werbung für Produkte und zum Abschöpfen von Daten genutzt, und sie bieten die technische Möglichkeit der Kontrolle all dieser Funktionen. Das Geschäftsmodell von Carsharing-Plattformen ist mit dem Verleih von Fahrzeugen allein ökonomisch nicht profitabel (Stehlin et al. 2020: 6). Das ökonomische Interesse zielt auf Netzwerkeffekte durch die Ausweitung der Dienste, auf Markendominanz durch die Sichtbarkeit neuer Fahrzeugmodelle in Großstädten und auf die Vermarktung von Beiprodukten (insbesondere personifizierter Bewegungsmuster).

Nick Srnicek theoretisiert diesen Prozess als Erster unter dem Begriff „platform capitalism“ (Srnicek 2016). Er bezeichnet damit die digitale Transformation des Kapitalismus seit der globalen Finanzkrise 2007/o8, die durch globale IT-Unternehmen vorangetriebene Entwicklung digitaler Plattformen als neues Geschäftsmodell sowie die daraus resultierende Unternehmensmacht. In der kritischen Plattformforschung wird auch der Begriff Technokapitalismus verwendet, um die sozialräumlichen Transformationen des urbanen Alltagslebens zu rekonstruieren (Sadowski 2020). Die Betreiber von intermediären Plattformen[4] versuchen, den auf Datenextraktivismus basierenden Plattform-Kapitalismus auch in der Branche der Mobilitätsdienstleistungen zu etablieren. Hier werden Kund_innen nicht nur als Nutzer_innen einer Dienstleistung betrachtet, sondern auch als Datenproduzent_innen. Insbesondere die Multimodalität im städtischen Verkehr bietet der Plattformökonomie vielfältig verwertbare Nutzer_innendaten. Die Anwendung der intermediären Plattformdienstleistungen ist in der Regel kostenlos (z. B. Google Maps), aber alle Nutzer_innenund Standortdaten können unter bestimmten Rahmenbedingungen des Datenschutzes von den Betreibern der Plattformen weiterverwertet und damit ökonomisch in Wert gesetzt werden. Mobilitätsplattformen werden damit zu Datenzulieferanten, und die Carsharing-Nutzer_innen optimieren 
die angebotenen Dienstleistungen durch ihre weiterverarbeiteten Daten quasi selbst (Daum 2018: 60).

Die zunehmende Plattformisierung (platformization) des Alltags wirft die Frage auf, wer für die Verankerung öffentlicher Werte in einer Plattformgesellschaft verantwortlich ist oder sein sollte. Dazu gehören individuelle Privatsphäre und Datensicherheit genauso wie die Berücksichtigung öffentlicher Interessen wie Fairness, Zugänglichkeit, demokratische Kontrolle, Transparenz und Rechenschaftspflicht. Um diese Werte konkurrieren in der Plattformgesellschaft Privatwirtschaft, Regierung und Zivilgesellschaft (van Dijck et al. 2018). Im Fall der europäischen Mobilitätsplattformen werden diese Werte fast ausschließlich von den Gesellschafts- und Zukunftsvorstellungen USamerikanischer IT-Konzerne und deutscher Automobilkonzerne vorgegeben.

Plattform-Urbanismus ist eine spezifische Entwicklung der Smart City - aber nicht mit ihr gleichzusetzen. Die frühere Phase des smart urbanism wurde noch top down in Public-private-Partnerships zwischen Stadtverwaltungen und IT-Konzernen wie Cisco und IBM vorangetrieben, die Software und digitale Infrastrukturen für kommunale Managementaufgaben (Verkehrssteuerung, Müllentsorgung etc.) installierten und in Kontrollzentren überwachten (Marvin et al. 2016). Der Plattform-Urbanismus führte nun zu einem stärker gouvernementalen Modus. Hier geht es um die Vermittlung von Dienstleistungen, die von Nutzer_innen aktiv für ihre Versorgungsbedarfe abgefragt werden und die indirekt Nutzer_inneninformationen in das System einspeisen. Einige Autor_innen sprechen mittlerweile von einer Plattformisierung, um den neuen Modus dieser Smart-City-Entwicklung zu kennzeichnen (Barns 2019; Stehlin et al. 2020; Lee et al. 2020). Ausgangspunkt der plattformvermittelten Dienstleistungen sind nicht Defizite der kommunalen Daseinsvorsorge, sondern ist die ökonomische Logik einer Diversifizierung des Angebots mit dem Ziel, eine zusätzliche Nachfrage zu generieren.

Im Bereich der Mobilität ist diese Plattformisierung spezifisch ausgeprägt und wird für Urbanisierungsprozesse in besonders relevanter Weise sichtbar, da ein konkretes Produkt zur zeitlich und räumlich begrenzten Nutzung vermittelt wird. Mobilitätsplattformen funktionieren anders als soziale oder Lernplattformen, die Informationen oder digitale Medien für beliebig viele Nutzer_innen zur Verfügung stellen, da sie eine materielle Infrastruktur an konkreten Orten für Einzelnutzer_innen verfügbar machen. Jede Plattform ist das Ergebnis von Aushandlungen verschiedener Akteure, die notwendige Teile der digitalen Infrastruktur bereitstellen und kontrollieren. In diesem integrierten System (vgl. Abb. 1) treffen die sektoralen Plattformbetreiber aus Automobilwirtschaft und öffentlichen Transportunternehmen mit der Digitalwirtschaft zusammen. Die digitalen Infrastrukturen materialisieren sich an unterschiedlichen Orten: in den Plattformservern, über das Internet in den digitalen Endgeräten (Smartphones), in den Identifikationssensoren der Fahrzeuge. Und mittels globaler Navigationssysteme (GPS) findet eine permanente Ortung der eingesetzten Endgeräte und Fahrzeuge statt.

Die Betreiberstrukturvon Mobilitätsplattformen stellt eine Übergangsform von traditionellen zu neuen Akteuren dar. Betrieben werden diese fast ausschließlich von Automobilkonzernen, die intermediäre Plattformen der ITKonzerne nutzen, um ihre Dienste anbieten zu können. Die aktuell größten 


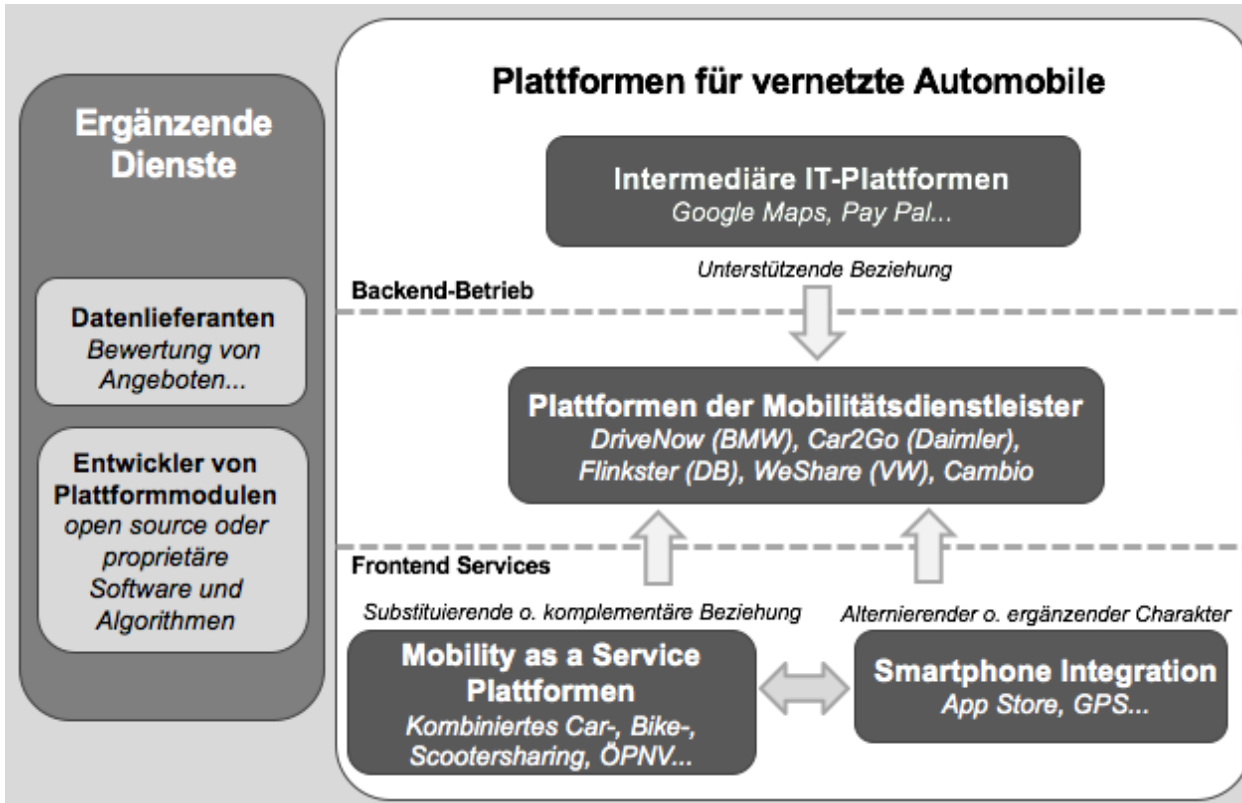

IT-Konzerne (Alphabet, Facebook, Amazon, Apple, Microsoft) haben über ihren Anteil an der Plattformsoftware und -infrastruktur in nur einem Jahrzehnt enorme Macht erlangt, die privaten und urbanen Lebensräume von Milliarden Menschen weltweit zu gestalten. Diese Macht ist mit einer geringen Rechenschaftspflicht gegenüber gesellschaftlichen Institutionen und einem geringen Interesse verbunden, in lokale Versorgungsbedarfe $\mathrm{zu}$ investieren. Mobilitätsplattformen sind auf diese Weise gleichzeitig in raumzeitliche Kontexte eingebettet und aus ihrer lokalen Verantwortung herausgelöst (Graham 2020: 2).

Die Ausprägung eines Plattform-Urbanismus ist nicht vorbestimmt oder alternativlos. Die neuen Formen von Konnektivität und Echtzeitdatenübermittlung können zur Ermöglichung progressiver Prozesse beitragen und gleichzeitig zu Problemen einer marktorientierten Stadtpolitik führen.

\section{Entwicklungslinien vernetzter Mobilität in Europa}

Smart Cities sind mit großen Versprechen hinsichtlich einer verbesserten Lebensqualität verbunden. Den Herausforderungen von Vielfachkrisen, die insbesondere Stadtgesellschaften treffen (Klimakrise, Verkehrskrise, CareKrise, Wohnungskrise), soll in der Vorstellung vieler Stadtregierungen und IT-Konzerne mit digitalen Steuerungstechnologien, Allzeitkonnektivität und Assistenzdienstleistungen begegnet werden. Mobilität spielt dabei eine besondere Rolle. Immer wieder aufgerufene Stichworte sind gesteigerte Ressourceneffizienz (weniger Fahrzeuge produzieren und teilen), Flexibilität (permanenter Zugang zu bedarfsgerechten Verkehrsmitteln), Optimierung (gesteuerter Verkehrsfluss) und Beschleunigung (Informationen über schnellste Verbindungen). Smart Mobility ist ein Bereich innerhalb des neuen Konzepts der Smart City, der ganz oben auf der europäischen Politik- und Forschungsagenda steht. Auch wenn der Nutzungsanteil von digital vermittelten Mobilitätsdienstleistungen im Vergleich zum öffentlichen Transport und zum motorisierten Individualverkehr noch sehr gering und bisher ein Großstadtphänomen ist (vgl. Bauriedl 2020), dient dieses neue Mobilitätsangebot vielen Stadtregierungen als sichtbarstes Smart-City-Produkt.
Endnutzer*in

Nutzung der Dienste im Auto

Installierte Dienste

via Smartphone Integration

Fernzugriff

Smartphone-App

Web-Portal

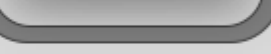

Abb. 1 Systematik intermediärer Plattformen, Online-Dienstleister und digitaler Kommunikationsmedien für die Nutzung von Mobilitätsplattformen (eigene Darstellung basierend auf Bosler et al. 2017) 
Die plattformvermittelte Mobilität ist von einer Vielzahl verschiedener Angebote geprägt (vgl. Tab. 1). Zentrale Unterscheidungsmerkmale sind neben der Stationsgebundenheit oder -ungebundenheit und dem Selbstfahren oder Chauffiertwerden die Art der institutionellen Einbettung sowie die Organisation der Dienstleistung. Beispiele für stationäre Carsharing-Netzwerke sind Cambio oder Flinkster. Als Peer-to-Peer-Plattformen (P2P-Plattformen) sind Snappcar und Getaround in Europa dominant. Allen Plattformen gemein ist die Nutzung von Plattformtechnologien zur Vermittlung der jeweiligen Dienstleistung. Dabei variieren die Verflechtungsintensität und die Informationsdichte digitaler Konnektivität bei den verschiedenen SharingFormen. Stationäre Carsharing-Angebote bedürfen meist keiner Datenvermittlung in Echtzeit und keiner permanenten Ortung der zur Verfügung stehenden Fahrzeuge. Diese Form des Carsharings ist in Deutschland bereits seit den 1980er Jahren verbreitet (Bauriedl 2020). Mobility-as-a-ServicePlattformen (MaaS-Plattformen) verknüpfen verschiedene Mobilitätsdienstleister mit anderen innovativen Diensten; sie verfügen über keine eigene physische Infrastruktur.

Beispielhaft für die oben genannten Mobilitätsdienstleistungen beschäftigen wir uns in diesem Artikel mit Angeboten des Free-Floating-Carsharing (FFC) und in Ergänzung mit dem Konzept Mobility as a Service (MaaS). Der Begriff Mobilitätsdienstleistung meint dabei als Oberbegriff alle Formen mobilitätsbezogener Dienstleistungen. FFC und MaaS beschreiben Unterkategorien und spezifische Formen der plattformvermittelten Mobilitätsdienstleistung. Im Folgenden betrachten wir die Praxis der dominanten Betreiber von FFC- und MaaS-Plattformen in Europa und lokal spezifische Ausnahmen in den jeweiligen Sektoren im Detail. Die Ausführungen stellen eine Momentaufnahme zum Zeitpunkt der Internetrecherche im Mai 2020 dar und stehen exemplarisch für einen ausdifferenzierten und im Umbruch befindlichen Mobilitätssektor.

Der Schwerpunkt der FFC-Angebote innerhalb Europas bezüglich der Zahl der Mobilitätsangebote und der Größe der Fahrzeugflotten liegt eindeutig in Deutschland. Dieser Markt wird momentan vor allem von der Plattform ShareNow geprägt, die von BMW und Daimler betrieben wird.

Tab. 1 Plattformvermittelte Sharing-Angebote im Mobilitätssektor (eigene Zusammenstellung) ShareNow wurde Anfang 2019 in einer Fusion aus DriveNow (BMW) und Car2Go (Daimler) gegründet, die schon seit 2011 beziehungsweise 2009 FFC anboten. Die Plattform bezeichnet sich selbst als ,ältester und bedeutendster Carsharing-Anbieter auf dem Markt“" (Share Now GmbH 2020). Aktuell (Mai 2020) befinden sich nach Selbstauskunft der Unternehmen

\begin{tabular}{|l|l|l|}
\hline Carsharing & stationär & $\begin{array}{l}\text { Das Fahrzeug kann an festen Stationen innerhalb der Stadt ausgeliehen und an der jeweiligen Station wieder } \\
\text { zurückgegeben werden. Im Falle des One-way-Carsharing kann das Fahrzeug auch an einer anderen Station } \\
\text { abgestellt werden. }\end{array}$ \\
\cline { 2 - 3 } & free floating & $\begin{array}{l}\text { Das Fahrzeug kann jederzeit an einem Standort ausgeliehen und an einem beliebigen Standort innerhalb des } \\
\text { Geschäftsbereichs des Anbieters abgestellt werden. Dasselbe Prinzip gilt in den meisten Fällen für Scooter-, } \\
\text { Roller- und Bike-Sharing. }\end{array}$ \\
\cline { 2 - 3 } & peer-to-peer & Privatpersonen vermieten das eigene Fahrzeug an andere Privatpersonen. \\
\hline Ridehailing & Privatpersonen bieten über eine App Fahrdienste mit dem eigenen Pkw an. \\
\hline Ridepooling & $\begin{array}{l}\text { Mehrere Personen mit Fahrtzielen in der gleichen Richtung teilen sich ein Fahrzeug mit Fahrer_in und Abhol- } \\
\text { service an der Straße. Die Strecke wird über einen Algorithmus berechnet. }\end{array}$ \\
\hline Mobility as a Service (MaaS) & $\begin{array}{l}\text { Über eine gemeinsame Plattform kann auf Mobilitätsplattformen verschiedener Anbieter zugegriffen werden, } \\
\text { die die effizienteste Fahrzeugkombination für die gewünschte Strecke zum Pauschalpreis berechnet. }\end{array}$ \\
\hline
\end{tabular}


rund 14.00o Fahrzeuge in acht europäischen Ländern im Einsatz. Neben dem Joint Venture von Daimler und BMW positionieren sich weitere Automobilhersteller auf dem Markt. Bei allen Anbietern ist der Anteil elektrisch betriebener Fahrzeuge sehr hoch und weiter wachsend (Bauriedl 2020). Die Plattform WeShare von Volkswagen bietet in Berlin ausschließlich elektrisch betriebene Fahrzeuge an. Nach der dortigen Implementierung der Carsharing-Plattform im Juni 2019 planen die Betreiber eine Einführung in sieben weiteren europäischen Städten (Volkswagen AG 2020). Weitere internationale Automobilhersteller auf dem europäischen FFC-Markt sind Renault mit der Plattform Zity in Paris und Madrid sowie die PSA Group (Peugeot, Citroën, Opel, DS Automobiles, Vauxhall) mit der Plattform free2move in Paris, Madrid und Lissabon (Groupe PSA 2017). Das schwedische Automobilunternehmen Volvo betreibt die Plattform „M“ in den Städten Stockholm, Göteborg und Malmö (Volvo Car Mobility 2020). Renault ist Partner der FFC-Plattform Green Mobility, die seit 2016 Carsharing-Dienste in Kopenhagen anbietet und seitdem schrittweise nach Oslo (2017), Malmö (2018) und zukünftig nach Göteborg expandiert (Green Mobility A/S 2020).

Neben Automobilherstellern sind zunehmend als Autovermietungen bekannte Unternehmen auf dem Carsharing-Markt aktiv. Die Autovermietung Sixt kündigte beispielsweise an, mit der Plattform Sixt Share den Unterschied zwischen Autovermietung und Carsharing auflösen zu wollen (Stüber 2019), und bietet seit Anfang 2019 in Berlin, Hamburg und München auch FFC an. Damit wird deutlich, dass sich Automobilhersteller und -dienstleister in Ergänzung zu ihrem Kerngeschäft auf dem FFC-Carsharing-Markt positionieren. Ein Gegenentwurf zu diesem Geschäftsmodell der Automobilkonzerne ist die Mobilitätsplattform Mobility in der Schweiz, die 1997 als Genossenschaft gegründet wurde. Sie bietet neben stationärem Carsharing in den Städten Basel und Genf auch eine Free-Floating-Option an (Mobility Genossenschaft 2020).

Analog zur Diversifizierung des FFC-Marktes nimmt seit etwa 2015 die Zahl der MaaS-Plattformen im Bereich der Mobilitätsdienstleistungen stetig zu. Die Plattformen, die unter dem Label MaaS gefasst werden, unterscheiden sich von Anbieter zu Anbieter und von Stadt zu Stadt in der Art der Bezahlung des Services, in der Zusammensetzung der Mobilitätsdienstleistungen und hinsichtlich der beteiligten öffentlichen und privaten Unternehmen. Vorreiter in der Entwicklung und Implementierung von MaaS-Plattformen ist das finnische Unternehmen MaaS Global. Die dazugehörige App „Whim“ bietet verschiedene Bezahlmöglichkeiten, vom Monats- bis zum Jahrestarif. Im gebuchten Zeitraum können Kund_innen alle im Zahlungsumfang enthaltenen Transportmittel teilweise unbegrenzt, teilweise zu den normalen oder reduzierten Konditionen des jeweiligen Anbieters buchen. Außer in Helsinki wurde die Plattform in den West Midlands in der Umgebung Birminghams, in Antwerpen und in Wien etabliert (MaaS Global Oy 2020). Ein weiteres Unternehmen auf dem europäischen MaaS-Markt ist Trafi. Es startete im Sommer 2019 in Berlin mit der App „Jelbi“ in Kooperation mit den Berliner Verkehrsbetrieben (BVG) die erste MaaS-Plattform in Deutschland. „Jelbi“ kombiniert Mobilitätsangebote des ÖPNV mit Roller-, Bike-, Scooter-, Car- und Ridesharing (Berliner Verkehrsbetriebe 2020). Basierend auf der Zieleingabe schlägt die Plattform-App eine geeignete Route vor. Alle Leistungen können mit einem einzigen digitalen Ticket über die App bezahlt werden. 
In der zusammenfassenden Betrachtung der Mobilitätsplattformen in Europa wird deutlich, dass sich die Angebote des FFC größtenteils auf die Großstädte beschränken, dass Automobilkonzerne und Autovermietungen an den meisten Plattformen beteiligt sind und durch Bereitstellung und Instandhaltung der Fahrzeugflotte deren Angebotsstruktur bestimmen. Grundsätzlich erstrecken sich die Geschäftsbereiche der Anbieter jeweils nur auf die Innenstadtbereiche. Die Stadtränder dürfen befahren, die Fahrzeuge dort aber nicht abgestellt werden.

Neben diesen grundlegenden Tendenzen kommt es zu Spezialisierungen und zur Vernetzung der verschiedenen Angebote untereinander. Diese Vernetzung erfolgt unter anderem in Form der beschriebenen MaaS-Plattformen, auf denen die jeweiligen Carsharing-Angebote nur einige von vielen Transportmöglichkeiten sind. Der deutsche FFC-Anbieter Miles ist Teil der „Jelbi“App. ShareNow kooperiert im Rahmen der „HVV Switch“-App in Hamburg mit anderen Carsharing-Anbietern, Taxiunternehmen und dem ÖPNV. Im Bereich der MaaS-Plattformen besteht durch die Kooperationen des kommunalen ÖPNV mit privaten Mobilitätsdienstleistern das Risiko, dass öffentliche Anbieter bestimmte Dienstleistungen an private auslagern und damit ihren Versorgungsauftrag vernachlässigen, zum Beispiel indem sie die Mobilitätsversorgung in peripheren Bereichen „Ridepooling“-Unternehmen wie Moia (VW) überlassen.

Wie oben beschrieben, sind Plattformen wie ShareNow momentan in mehreren europäischen Städten mit denselben Dienstleistungen vertreten. WeShare bewegt sich in eine ähnliche Richtung, indem das Unternehmen in weitere Städte expandieren will. Damit wird ein standardisiertes Geschäftsmodell in Städten mit unterschiedlicher lokaler Struktur implementiert, ohne auf vielfältige Gegebenheiten und Bedarfe eingehen zu können. Ein Grund dafür kann die Bindung von Kund_innen sein, die Carsharing in unterschiedlichen Städten nutzen. Das einheitliche Design schafft einen Wiedererkennungswert auch an anderen Standorten.

Sektorale Plattformen sind auf zweierlei Weise mit anderen sektoralen oder intermediären Plattformen vernetzt (vgl. Abb. 1): zum einen über die oben beschriebene Bündelung in MaaS-Apps. Zum anderen greifen die Plattformen zu großen Teilen auf dieselben intermediären Plattformen wie Google Maps oder PayPal zurück. Stehlin, Hodson und McMeekin beschreiben Plattformen im Unterschied zu materiellen Infrastrukturen der Vermittlung von Gütern und Dienstleistungen als eher fragil (Stehlin et al. 2020: 14). Dennoch folgen Automobilhersteller und andere Mobilitätsdienstleister dieser Entwicklung, um eine autogerechte Stadtentwicklung auch in Zeiten der Plattformisierung fortführen zu können oder um ihre dominante Stellung in den neuen Märkten der Mobilitätsdienstleistungen zu wahren.

\section{Probleme des Zugangs zu Smart-Mobility-Angeboten}

Die Debatte um den aufkommenden Plattform-Urbanismus orientiert sich oftmals ausschließlich an den damit einhergehenden technischen Möglichkeiten und Zusammenhängen (Lee et al. 2020; Stehlin et al. 2020). Dies zeigt sich auch anhand des Mobilitätssektors und der damit verknüpften Angebote und Entwicklungen im Bereich Smart Mobility. Das hohe 
Tempo der technologischen Entwicklung (insbesondere bei Sensortechnik, Datengeschwindigkeit, Speicherkapazitäten und Konnektivität) und die Notwendigkeit, infolge der Klimakrise neue Formen urbaner Mobilität zu entwickeln, geht in den meisten Städten mit einer stark technologieoptimistischen Verkehrspolitik einher. Die Mobilitätsdebatte ist in großen Teilen von Aussagen zu den positiven Potenzialen technologischer Innovationen geprägt (Liyanage et al. 2019) und verweist auf Studien zur Effizienzsteigerung durch digitale Technologien, die zu einem nachhaltigeren Umgang mit begrenzt verfügbaren Ressourcen verhelfen soll (Rid et al. 2018). Im Vergleich zu den versprochenen großen Effekten einer ökologischen Transformation (Ressourceneinsparung) werden die notwendigen sozialen Transformationen (gerechter Ressourcenzugang) vernachlässigt.

Zudem wird der Einfluss neuer Mobilitätsangebote vor dem Hintergrund einer potenziellen gesellschaftlichen Abkehr von individueller Automobilität diskutiert (Groth 2019c; Kent/Dowling 2013). Wir folgen in diesem Aufsatz der von Sheller und Urry (2006) ausgerufenen „Mobilitätswende“ in den Sozialwissenschaften. Diese hat nicht nur zu einem neuen Verständnis von Verkehrsströmen (konventionell wie virtuell) geführt, sondern darüber hinaus die Mobilitätsforschung für qualitative Zugänge und interpretative Ansätze geöffnet. Mobilitätskontexte werden nun intensiver als Orte der Aushandlung sozialer Verhältnisse und Konflikte untersucht; damit ist die Voraussetzung für eine theoretische Auseinandersetzung mit den Spannungsfeldern urbaner Mobilität geschaffen.

Im Folgenden setzen wir im Anschluss an diesen Perspektivwechsel die im vorausgegangenen Abschnitt beschriebenen diversifizierten technologischen Entwicklungen in Bezug zu realer und potenzieller sozialer Ausgrenzung und fragen, wie sich Formen der Ausgrenzung konzeptionell fassen lassen und welche Konfliktlinien dabei in Erscheinung treten. Unser Argument fokussieren wir auf Konzepte, die am Schnittpunkt zwischen Mobilität und sozialer Ausgrenzung in europäischen Städten verortet werden können. Konzepte mit Blick auf Potenziale neuer Technologien (Kenyon et al. 2002) oder die Bedeutung von Smart Mobility für die Konnektivität ländlicher Räume (Velaga et al. 2012) können an dieser Stelle nicht im Detail diskutiert werden. Auch Studien zu Smart Mobility in den USA, die zum Beispiel Einstellungsmuster zu neuen emissionsfreien Carsharing-Fahrzeugen (Shaheen et al. 2020) untersuchen, haben wir nicht mit einbezogen, da sich Mobilitätsnachfrage und -kultur sehr von der Dynamik in europäischen Großstädten unterscheiden.

Mobilitätsinfrastrukturen sind ein Ergebnis gesellschaftlicher Auseinandersetzungen in der Vergangenheit, und sie bestimmen die Möglichkeiten der Mobilitätsteilhabe in Gegenwart und Zukunft. Church et al. (2000: 198 ff.) fassen Formen der Ausgrenzung basierend auf verschiedenen Charakteristika des Mobilitätssektors zusammen und bieten damit einen konzeptionellen Rahmen für den Zusammenhang zwischen sozialer Ausgrenzung und Mobilität:

1. physische Ausgrenzung: zum Beispiel durch das Design des Fahrzeugs;

2. geographische Ausgrenzung: etwa durch die Wohnlage im suburbanen oder ländlich-peripheren Raum und die Zerschneidung der Stadt durch Schnellstraßen; 
3. Ausgrenzung von zentralen Einrichtungen: So kann etwa der Zugang zu Schulen oder Gesundheitseinrichtungen durch große Entfernungen zu diesen erschwert werden;

4. ökonomische Ausgrenzung: zum Beispiel durch hohe fixe und/oder flexible Mobilitätskosten;

5. zeitbasierte Ausgrenzung: etwa der Zeitmangel für Mobilität (time poverty) verursacht durch die Doppelbelastung von Lohn- und Care-Arbeit;

6. Ausgrenzung durch Angst: zum Beispiel bei Sicherheitsbedenken und/oder Gewalterfahrungen im Zusammenhang mit spezifischen Transportmitteln oder -wegen;

7. räumliche Ausgrenzung: zum Beispiel durch Gated Communities.

Den einzelnen Ausgrenzungsaspekten kommt je nach gesellschaftlichem Kontext und sozioökonomischer Positionierung der jeweiligen Person oder Personengruppe verschieden große Bedeutung zu. Außerdem können sich diese Ausgrenzungserfahrungen und -praktiken überlagern und dadurch für spezifische Situationen, Tageszeiten oder Räume potenzieren. Auch wenn die Ausführungen von Church et al. (2000) schon vor zwei Jahrzehnten veröffentlicht worden sind, bleiben die damit verbundenen Forderungen einer Stärkung des öffentlichen Nahverkehrs und einer lokal spezifischen und qualitativ informierten Behandlung der Probleme mobilitätsbezogener Ausgrenzung auch heute relevant (vgl. ebd.: 203 f.).

Aufbauend auf der konzeptionellen Rahmung von Church et al. (2000) hat Karen Lucas (2012) zur Beschreibung des Zusammenhangs von sozialer Ausgrenzung und Mobilität den Begriff der „transport poverty“ (ebd.: 107) geprägt. Laut Lucas überlagern sich entlang der Ausgrenzungsdimensionen verschiedene Formen mobilitätsbezogener Benachteiligung (z. B. der Mangel an Information über Mobilitätsmöglichkeiten oder hohe Transportkosten) mit Aspekten sozialer Benachteiligung (z. B. geringes Einkommen oder mobilitätsbeeinträchtigender Gesundheitszustand) (ebd.). Die Mobilitätseinschränkung kann wiederum mangelnde Zugänge zu politischen Beteiligungsprozessen, sozialen Netzwerken oder beruflichen Entwicklungsmöglichkeiten zur Folge haben und damit zu sozialer Ausgrenzung führen. Lucas betont somit den Anspruch an digitale Mobilitätsplattformen und neue Mobilitätsangebote, das Problem der „transport poverty“ zu berücksichtigen (ebd.: 108). Die Frage ist, ob und inwieweit FFC- und MaaS-Angebote zur Abschwächung der genannten Ausgrenzungen dienen können.

Sören Groth (2019a) beschreibt eine Veränderung, Verschärfung und Reproduktion der Formen mobilitätsbezogener sozialer Ausgrenzung infolge zunehmender Digitalisierung am Konzept des multimodal divide. Er argumentiert, dass den Personen, die von transport poverty betroffen sind, neben den oben beschriebenen gesellschaftlichen Teilhabemöglichkeiten auch die „digitalen Möglichkeiten zur Ausübung eines multimodalen Verhaltens auf der Basis vernetzter Mobilitätsdienstleistungen“ fehlen (ebd.: 25). Das Konzept ist im Anschluss an den Begriff digital divide zu verstehen, der die Spaltung der Gesellschaft entlang der kognitiven, finanziellen und technologischen Möglichkeiten zur digitalen Teilhabe beschreibt. Groth nennt das Beispiel der infrastrukturellen Unterversorgung ländlicher Regionen (z. B. mit 
Breitband-Internetzugang), die die gesellschaftliche Teilhabe an einer digitalen Transformation der dort lebenden Bevölkerung einschränkt (Groth 2019b: 61). Auf individueller Ebene benennt er drei spezifische Formen, in denen sich diese Ausgrenzung im Sinne eines multimodal divide manifestiert:

1. Die Teilhabe an vernetzten Mobilitätsdienstleistungen ist voraussetzungsvoll. Neben dem Besitz eines Smartphones sind auch Zugang zu und Kenntnis von Ressourcen wie der Nutzung einer Kreditkarte oder der Verfügung über ein Bankkonto sowie eine Meldeadresse Bedingung. Diese Wissensbestände und materiellen wie institutionellen Zugänge sind gesellschaftlich sehr ungleich verteilt. Die Wissensbestände im Umgang mit digitalen Technologien weisen außerdem eine deutliche Generationenkluft auf.

2. Plattformbasierte Mobilitätsdienstleistungen sind auf eine technik- und digitalisierungsaffine, erwerbstätige Personengruppe ausgerichtet. Kate Pangbourne et al. (2020: 44) beschreiben diese als „urbane Elite, die es sich leisten kann".

3. Die Teilhabe an den beschriebenen Plattformen bedarf einer bereitwilligen Freigabe von persönlichen Daten bei der Registrierung und gewährt dem Betreiber der intermediären Plattform damit - im Rahmen mehr oder weniger weitreichender Regulierungen - den personalisierten Zugriff auf Bewegungsdaten mittels permanenter Ortung in der jeweiligen Anwendung.

Datensouveränität ist ein nicht zu unterschätzender Ausgrenzungsaspekt im Plattform-Urbanismus. Nutzer_innen der Angebote sind nicht nur Konsument_innen einer Mobilitätsdienstleistung, sondern auch Produzent_innen von Bewegungsdaten, die zur Optimierung der Plattformdienste verwendet werden. Sie sind gezwungen, sich auf den gouvernementalen Modus der Plattformbetreiber einzulassen (Stehlin et al. 2020). Auf diese Weise werden kategorisch jene Menschen von der Nutzung der Angebote ausgeschlossen, die sich aus politischen, technologiekritischen oder sonstigen Gründen diesem Zugriff verweigern.

\section{Genderbezogene Kluft der Smart-Mobility-Praxis}

Mobilität ist nicht geschlechtsneutral, da Geschlechterverhältnisse und Mobilitätsstrukturen auf tiefgreifende und oft subtile Weise miteinander verbunden sind. Mobilität als gesellschaftliche und räumliche Praxis und insbesondere die Kultur der Automobilität in Industrieländern sind über Jahrzehnte vergeschlechtlicht (Uteng/Cresswell 2008) und rassifiziert (Sheller 2015) durch Repräsentationen, Bedeutungen und Praktiken. Diese Feststellung gilt auch für Smart Mobility. Die Idee von Smart Mobility ist eng verknüpft mit dem Versprechen effizienter genutzter Fahrzeuge und einem optimierten Verkehrsstrom. Damit lässt sich auch die Idee einer „autogerechten Stadt“ fortsetzen, die die gegenwärtige europäische Stadt seit den 1960er Jahren geprägt und zu sozial segregierten Städten und vergeschlechtlichter Mobilität geführt hat. Reproduzieren Carsharing und Mobilitätsplattformen diese Ungleichheitsstrukturen und führen auf diese Weise zu einem gendered platform urbanism? 
Die vorliegenden Analysen zur Carsharing-Nutzung (die sich entweder auf die Zahl der Registrierungen auf den Mobilitätsplattformen oder auf Ausleihvorgänge beziehen) zeigen deutlich, dass soziodemografische Variablen hier eine wichtige Rolle spielen. Schlüsselvariablen sind in den meisten Studien Geschlecht, Alter, Einkommen und Bildungsstatus. In allen Studien verfügen die Nutzer_innen von Carsharing über einen hohen Ausbildungsgrad und ein überdurchschnittliches Einkommen, und sie leben häufig allein. Die Nutzer_innen von stationärem Carsharing sind durchschnittlich älter als die Nutzer_innen von FFC. Frühe Studien aus den USA (insbesondere aus San Francisco um 2005) zeigen einen auffällig höheren Anteil von Frauen als Hauptnutzer_innen, was mit der Bedeutung des Autobesitzes als maskulinem Statussymbol erklärt wird (Hildebrand/Sheller 2018). In chinesischen Städten nutzen eher Männer und ältere Menschen Carsharing; Nutzungsmotive sind vor allem Einkaufs- und Freizeitfahrten.

Studien zu europäischen Städten zeigen wiederum ein anderes Bild: Hier sind eindeutig Männer die Hauptnutzer_innen und die Nutzung wird stärker von den Kosten und dem Zugang zu Parkplätzen motiviert als vom Wegezweck (Alonso-Almeida 2019: 38). Quantitative Studien für verschiedene deutsche Großstädte zeigen, dass FFC-Nutzer_innen bis zu 8o Prozent männlich, überdurchschnittlich gut gebildet und vollzeitberufstätig sind (Giesel/Nobis 2016). Zu vielen Nutzungsaspekten, die für Aussagen zur sozialräumlichen Privilegierung oder Exklusion relevant wären, liegen allerdings keine Daten vor. Etwa, welche Bedingungen gegebenenfalls gegen die Registrierung auf einer Mobilitätsplattform sprechen oder ob es signifikante räumliche Schwerpunkte der Wohnorte der Nutzer_innen gibt (Innenstadt, Stadtrand oder Umland, in größerer oder geringerer Entfernung vom Nutzungsort).

Feministische Mobilitätsforschung untersucht seit den 1970er Jahren Ausschlussfaktoren von Mobilität - also eher Immobilität. Im Vordergrund des Forschungsinteresses steht dabei der Aspekt sozialer Ermächtigung durch Mobilität, nicht etwa beschleunigte oder effizientere Distanzüberwindung. Mobilität stellt eine Möglichkeit dar, das Haus zu verlassen und in die Öffentlichkeit zu treten; die Einschränkung von Mobilität ist immer auch eine Beschränkung sozialer und gegebenenfalls ökonomischer Teilhabe. Zentral war und ist die Frage, wie die Mobilität(-smöglichkeit) Geschlechterverhältnisse und Geschlechtsidentität prägt und wie dies in patriarchale Machtverhältnisse eingebettet ist (Hanson 2010: 8).

Fast alle vorliegenden Mobilitätsstudien betrachten Geschlechterverhältnisse aus einer differenztheoretischen Perspektive. Darin werden immer wieder vier Ungleichheitsstrukturen als entscheidend für vergeschlechtlichte Mobilitätsformen hervorgehoben:

1. Geschlechtliche Arbeitsteilung, die durch einen höheren Frauenanteil in der Teilzeitarbeit und im Niedriglohnsektor mehr Kurzstreckenmobilität von Frauen zur Folge hat. Zudem führt die stärkere Belastung mit unbezahlter Sorgearbeit zu einem höheren Frauenanteil bei der Versorgungsmobilität, die mit komplexen Wegeketten und mehr multimodaler Mobilität von Frauen verbunden ist. Mehrheitlich in Vollzeit lohnarbeitende Männer verfolgen größtenteils Start/Ziel-Wege zu Hauptverkehrszeiten mit längeren Wegstrecken (Uteng 2011); 
2. sexistische Belästigungen gegenüber Frauen, die ein besonderes tageszeitspezifisches Sicherheitsbedürfnis von Frauen im öffentlichen Raum und die Vermeidung von als gefährdend empfundenen Mobilitätsformen zur Folge haben; [5]

3. vergeschlechtlichter Status von Verkehrsmitteln, der Automobilität mit männlicher Freiheit, Unabhängigkeit und Schutz der Familie assoziiert und große, schnelle Autos eher mit Männlichkeit und kleine, praktische Autos eher mit Weiblichkeit konnotiert;

4. geschlechtliche Einkommensunterschiede, die die hohen Anschaffungskosten von Privatfahrzeugen eher für Männer ermöglichen und Frauen eher auf den öffentlichen Transport oder nicht-motorisierte Mobilität angewiesen machen, die keine fixen Kosten verursachen, aber höhere flexible Kosten erzeugen.

Diese Faktoren verstärken sich gegenseitig. Die eingeschränkte Verkehrsmittelwahl von Frauen hat zum Beispiel einen höheren Zeitaufwand durch langsamere Verkehrsmittel zur Folge (transport poverty).

Neben dem Fokus auf Mobilitätsbarrieren hat sich die feministische Forschung seit den 1990er Jahren mit der Hierarchisierung von Mobilitätsformen beschäftigt. Dem automobilen Subjekt wird in der Positionierung mobiler Subjekte in der Stadtplanung und der stadtpolitischen Debatte seit Jahrzehnten der höchste Status eingeräumt. Es steht in Konkurrenz zu Fußgänger_innen, Benutzer_innen des öffentlichen Verkehrs, Radfahrer_innen und motorisierten Mikromobilist_innen, denen damit eine von der dominanten sozialen Norm abweichende und im Straßenraum störende Position zugewiesen wird. Abzulesen ist dieses Verhältnis auch in der Prioritätensetzung der Raumplanung bei der Gestaltung des öffentlichen Raums und der kommunalen Budgets für Fuß- und Radwege im Vergleich zur Subventionierung von Straßen- und Parkraum für Pkw. Durch Carsharing wird es vielen Menschen möglich, den Status eines automobilen Subjekts zu erlangen, da die hohen Anschaffungskosten entfallen und die Verfügbarkeit von Autos erhöht wird. Gleichzeitig schränkt dieses zusätzliche Automobilangebot den Nutzungsraum für nicht-motorisierte Mobilität ein.

Obwohl Smart-Mobility-Plattformen noch ein relativ junges Phänomen sind, liegen hierzu schon einige Arbeiten aus Perspektive der Geschlechterforschung vor (vgl. den Sammelband Gendering Smart Mobilities von Uteng et al. 2019). Die Beiträge befassen sich vor allem mit der Implementierung von Geschlechtergerechtigkeit in der Mobilitätsforschung und Planungspraxis. Zudem kritisieren sie die binären Geschlechterkategorien („männlich“/,,weiblich“) der meisten Mobilitätsstudien, da diese fast ausschließlich auf quantitativen Erhebungen basieren und sich Aussagen zu normativen Setzungen von Smart-Mobility-Dienstleistungen sowie eine Privilegierung von männlich konnotierten Mobilitätsformen nicht differenziert verifizieren lassen.

In ihrem Literaturreview zur Smart-Mobility-Forschung fragt Yamini Singh: „Is smart mobility also gender-smart?“ (2019), und kommt zu dem Schluss, dass es zu einer positiven Antwort wenig Anlass gebe und dies auch gar nicht die Intention von Smart-Mobility-Angeboten sei. Zum Geschäftsmodell von Smart-Mobility-Plattformen gehört, wie bereits ausgeführt, 
das Abschöpfen von Bewegungsprofilen der Nutzer_innen, auf die weitere Angebote abgestimmt werden. Damit tritt ein sich selbst verstärkender Prozess ein, der allein spezifische soziale Gruppen und urbane Identitäten sowie deren Mobilitätsbedürfnisse anspricht. Solange die Bewegungsmuster etwa von Sorgearbeiter_innen unsichtbar bleiben und Carsharing nach bewährtem Muster angeboten wird, verstärkt sich der gendered digital gap (Strüver/Bauriedl 2020; Alonso-Almeida 2019: 38).

Das Ideal einer ,Mobilität für alle und die formulierte Aufmerksamkeit für diverse urbane Identitäten und Lebensqualitäten in Smart-Mobility-Kampagnen erscheinen eher als Dekoration und als weiche Faktoren der technologischen Fixierung auf Konnektivität und Automatisierung (Balkmar/ Mellström 2018). In Imagekampagnen von Carsharing-Anbietern werden Frauen, Kinder und ältere Menschen auf den Passagiersitz verbannt, falls sie überhaupt an Bord genommen werden.

Weder FFC- noch MaaS-Plattformen sind für die Verkettung von Wegen zum Arbeitsplatz, Arztbesuch, Schule, Kindergarten, Einkauf und anderen Zielen eingerichtet. Gleichzeitig erhält mit der Priorisierung von SmartMobility-Plattformen der öffentliche Nahverkehr Konkurrenz, und so wird gegebenenfalls der Ausbau dieses Mobilitätsangebots gebremst, das aktuell stärker von Frauen und Menschen mit geringeren Einkommen genutzt wird.

\section{Schlussfolgerungen}

In unserer Analyse zur sozialen und räumlichen Differenzproduktion durch Plattformen und digitalisierte Mobilitätsdienstleistungen, diemittlerweilein fast allen Großstädten Europas und in besonderem Umfang in deutschen Städten angeboten werden, ist eine Plattformisierung der Stadtentwicklung deutlich geworden. Diese Plattformisierung resultiert aus dem Zusammenwirken soziotechnischer und politisch-ökonomischer Transformationen, die zu einer wachsenden Gestaltungsmacht von Automobil- und IT-Konzernen in Städten geführt hat. Aus unserer Sicht lassen sich fünf zentrale Prozesse des platform urbanism identifizieren, die eine sehr spezifische Wahrnehmung von Städten und bestimmte Vorstellungen von Stadtzukunft vonseiten der Plattformbetreiber und Smart-City-Protagonist_innen offenlegen:

\section{,Stadt ohne Ränder':}

Selektive Raumwahrnehmung von Plattformunternehmen

Smart-Mobility-Dienstleistungen sind nicht nur selektiv auf supervernetzte Menschen ausgerichtet, sie sind auch räumlich selektiv. Die Angebote sind auf Innenstadtquartiere in Städten mit über 300.00o Einwohner_innen beschränkt. Diese räumliche Konzentration ist nicht durch fehlende Netzkapazitäten oder unzureichende Verkehrsinfrastruktur im suburbanen Raum begründet, sondern allein durch die ökonomische Logik der privaten Mobilitätsanbieter: In den Zentren von Großstädten finden sie die favorisierten Kund_innen beziehungsweise Datenproduzent_innen und die gewünschte Aufmerksamkeit für ihre Produkte (Alonso-Almeida 2019; Bauriedl 2020). Das heißt: Carsharing verfolgt nicht primär das Ziel, Lösungen für sozialräumliche Ungleichheiten und Mobilitätsdefizite zu bieten. 
Carsharing könnte ein alternatives Transportmittel bieten - als ergänzendes Mobilitätssystem, das in Stadtteilen eingesetzt wird, in denen der öffentliche Transport nicht zuverlässig, effizient oder erreichbar ist. Die kurzen Wege des innerstädtischen Verkehrs können am umweltgerechtesten, sichersten und gesündesten über ÖPNV und nicht-motorisierte Mobilität bewältigt werden. Die MaaS-Angebote haben durch die Kombination mit dem öffentlichen Transport zwar eine größere Reichweite, lassen aber komplexe Wegeketten von Lohn- und Sorgearbeit schwer realisieren. Die Angebote sind auf räumlich-lineare Mobilitätsbedarfe mit Start- und Zielpunkten ausgerichtet und somit auch geschlechtlich selektiv. Die Mobilitätsmuster insbesondere von vielen Frauen, die Lohn- und Sorgearbeit kombinieren oder von den suburbanen Siedlungsräumen mit fehlendem Anschluss an den öffentlichen Nahverkehr ins Zentrum gelangen wollen, werden nicht bedient. Durch diese räumliche Struktur der Reichweite und Streckenberechnung für Einfachziele wird eine vergeschlechtlichte Segregation verstärkt.

\section{,Stadt als Folie؛:}

\section{Standardisierung der Stadtzukunft durch Mobilitätsplattformen}

Die Plattformisierung von städtischer Mobilität ist aus zwei Gründen immer mit Standardisierungseffekten verbunden: Erstens beruht die Logik von intermediären Plattformen auf Kommunikations- und Vernetzungsstandards und standardisierten Schnittstellen, und zweitens werden die Mobilitätsangebote der sektoralen Plattformbetreiber von den gleichen Akteuren weitergetragen, die auch schon die Stadtentwicklung der vergangenen Jahrzehnte dominiert haben - autoaffine Stadtplaner_innen und Automobilkonzerne. Diese gehen davon aus, dass die Stadtgesellschaften der Gegenwart noch die gleichen Anforderungen an eine urbane Mobilität stellen wie in den Anfängen der autogerechten Stadt der 1960er Jahre. Diese Standardangebote treffen auf eine stark diversifizierte Gesellschaft, deren Ansprüche an Urbanität, soziale Dichte, Stadtkultur und Zugänglichkeit des öffentlichen Raums sich stark gewandelt haben. Die europäischen Smart Cities bleiben jedoch mit der Präferenz für Carsharing, Ridepooling und perspektivisch mit dem vollautomatisierten Fahren auf dem Pfad der, autogerechten Stadt' und schaffen eine räumliche Fixierung von digitalen Mobilitätsinfrastrukturen (Sensoren für Parkraummanagement und autonomes Fahren).

\section{,Stadt als Ort ohne Eigenschaften':}

\section{Universalisierung der Plattformdienstleistungen}

In den meisten europäischen Städten agieren dieselben Betreiber von Mobilitätsplattformen. Sie verfolgen für alle Standorte der angebotenen Plattformdienstleistungen dieselben Vorstellungen von Stadtzukunft und Stadtgestaltung und bieten entsprechende Universallösungen für alle Mobilitätsanforderungen an, ohne lokal gewachsene Strukturen und Bedarfe zu berücksichtigen. Diese Ausblendung urbaner Diversität folgt der unternehmerischen Logik, durch Angebote, die schon aus anderen Städten bekannt sind, eine Nachfrage zu generieren (vgl. die Verbreitung von Scootern). Aufgrund der in Abschnitt 3 beschriebenen konstanten Ausweitung der 
Dienstleistungen internationaler Mobilitätsplattformen ist davon auszugehen, dass sich die Tendenz der Universalisierung verstärken wird.

Wettbewerbsorientierte Stadtregierungen haben eher ihre Platzierung auf internationalen Smart-City-Rankings im Blick als lokale Probleme der Mobilitätsversorgung und lokalspezifische Sicherheitsdefizite. Mobilitätsplattformen und Apps werden nicht politisch gefördert und subventioniert, um diverse und heterogene Mobilitätsinteressen gestaltbar zu machen und zu bedienen, sondern um globalisierten Digitalisierungs- und Mobilitätsvorstellungen zu folgen. Paradoxerweise wachsen das Carsharing-Angebot und damit der Einfluss der Plattformen auf urbane Mobilität genau an den Orten, wo es hervorragende öffentliche Verkehrsmittel und gute Radfahrmöglichkeiten gibt und oft schon andere Carsharing-Angebote zu finden sind.

,Stadt(-gesellschaft) ohne Vielfalt':

Normierung von urbanen Identitäten durch Plattformen

Plattformen sind keine sozial neutralen Mobilitätsermöglicher. Die verwendeten Algorithmen implizieren Normen und Werte, und das Angebot adressiert bewusst spezifische Bevölkerungsgruppen. Mobilitätsbedarfe von Sorgeleistenden werden durch Smart-Mobility-Dienstleistungen nicht explizit bedient. Weder die angebotenen Fahrzeuge noch die offerierten Dienstleistungen sind darauf ausgerichtet, verschiedene Ziele und Zwischenaufenthalte in einem Leihvorgang zu kombinieren und dabei Menschen mit speziellen Bedarfen oder Dinge im größeren Umfang zu transportieren. Diese für Sorgeleistende übliche Praxis erscheint als nicht-normales Mobilitätsverhalten für Plattformbetreiber und wird dem öffentlichen Nahverkehr überlassen.

,Stadt als Utopie:

Reproduktion der Zukunftsvisionen der Moderne

In den (vielfach visualisierten) Visionen der Smart City zeigen sich Ideen der Moderne mit digitaler Konnektivität zwischen Menschen und ,smarter Infrastruktur im städtischen Raum - einschließlich seiner vergeschlechtlichten Formation. In der damit verbundenen Materialisierung wird das Automobil zur Hülle eines Multimediaraums. Smart-City-Lobbyist_innen betrachten dabei FFC nur als Zwischenstufe zum selbstfahrenden, fahrerlosen Automobil beziehungsweise zum autonomen Fahren. Das ,Verhalten“ des Autos soll zukünftig vollständig von Algorithmen und mit Künstlicher Intelligenz erlernten Erfahrungswerten bestimmt sein und nicht die individuellen Gefühle und Bedürfnisse der Mitfahrenden nach Sicherheit, Wohlbefinden oder Geschwindigkeit berücksichtigen. Werden die Standardisierungen und Normierungen der aktuell installierten Plattformen weitergeführt, wird diese Mobilitätszukunft den aktuellen (generational and gendered) digital divide verstärken.

Mit unserem Befund, dass Smart-Mobility-Plattformen soziale Ungleichheit in Städten eher verschärfen als ausgleichen, befinden wir uns in guter Gesellschaft. Kritische Stadtforscher_innen üben seit einigen Jahren eine fundamentale Kritik an einer algorithmenbasierten und durch IT-Konzerne geprägten 
Stadtentwicklung. Dies ist jedoch nicht per se digitalisierungsfeindlich oder technikdystopisch. Die Autor_innen kritisieren die Priorisierung einer technologischen Transformation ohne explizite Strategien für eine sozial gerechte Transformation und betonen die emanzipatorischen Potenziale in digitalen Raumpraktiken. Rob Kitchin (2018: 2) folgert aus seiner Kritik des smarturbanism, dass es notwendig sei, sich Smart Cities neu vorzustellen, neu zu rahmen und neu zu gestalten (,reimagine, reframe and remake smart cities“), um Urbanisierung mit Fairness, Gerechtigkeit, Ethik und Demokratie verbinden zu können. Mark Graham (2020: 3) führt diesen Ansatz mit einer praktischen Anleitung für einen platform urbanism unter den Stichworten ,,regulate, replicate, resist" weiter. Er schlägt vor, Plattformen lokal reguliert einzubetten. Nur so könnten Datensouveränität und eine Kontrolle über die Nutzungsgebühren der Dienstleistungen erlangt werden. Eine demokratische Gestaltungsmacht über räumlich und sozial selektive Informationen der Plattformen ist nur über Open-Source-basierte Plattformen zu gewährleisten, die durch kommunale Kooperation und Schwarmintelligenz (weiter-)entwickelt werden.

Eine nachhaltige digitale Transformation sollte einem Gemeinwohlideal folgen, das von den Bedürfnissen und Fähigkeiten der Bewohner_innen der Stadt ausgeht und erst danach die verfügbaren Technologien sucht (WBGU 2019: 1). Sie müsste gekennzeichnet sein durch eine Stärkung zivilgesellschaftlicher Partizipation an Stadtgestaltungsprozessen und eine Minimierung digitaler Segregation (digital divide) innerhalb der Städte und der Stadt-Land-Beziehungen sowie zwischen Gruppen unterschiedlicher Generationen, Geschlechter, Einkommensverhältnisse und Herkunft.

Für das Beispiel geschlechtergerechter Smart-Mobility-Dienstleistungen könnte eine nachhaltige Transformation alternativ auch auf Suffizienz anstatt Ressourceneffizienz und Optimierung basieren: Technologien einer digitalen Konnektivität können genauso gut dazu genutzt werden, motorisierten Verkehr aus der Stadt zu halten und Fußverkehr sowie kostenfreien öffentlichen Transport zu befördern, um den Straßenraum als gesunden, sicheren und gemeinschaftlich geteilten öffentlichen Raum nutzen zu können. Aufgrund der aktuellen Krisen infolge der Coronapandemie und des Klimawandels ist schwer absehbar, in welcher Geschwindigkeit sich ein plattformvermitteltes Carsharing ausbreitet oder gar vom vollautomatisierten Fahren überholt wird. Die politischen Entscheidungen für oder gegen eine Subventionierung der Automobilwirtschaft, für oder gegen einen deregulierten Plattformkapitalismus und für oder gegen einen vergrößerten digital divide werden gegenwärtig getroffen und damit die Potenziale von Mobilität und urbaner Transformation festgelegt.

Dieser Artikel wurde durch die Universität Graz im Elisabeth-ListFellowship „Geschlechtergerechtigkeit und Smart Cities“ gefördert. 


\section{Endnoten}

[1] Die oft synonym verwendeten Begriffe Mobilität, Transport und Verkehr implizieren unterschiedliche sozialräumliche Komplexität. Transport und Verkehr bezeichnen die Bewegung von Menschen in einem instrumentellen Verständnis (Messung von Zeit, Distanz, Kosten). In der kritischen Stadtforschung wird seit dem cultural turn um die Jahrtausendwende der Begriff Alltagsmobilität verwendet, um die neue Einbettung des Themas in soziale und kulturelle Mobilitätsgeographien zu signalisieren (Law 1999). In diesem Beitrag verwenden wir „Mobilität“ explizit für (analoge) Alltagsmobilität mit Wegezwecken für Arbeit, Freizeit, Geselligkeit und Versorgung (und explizit nicht als Bezeichnung für virtuelle Mobilität, soziale Mobilität oder Migration).

[2] Dieser Text wurde geschrieben, während kurzzeitig die Produktionsbänder deutscher Automobilkonzerne stillstanden, der städtische Verkehr aufgrund von Kurzarbeit, Homeoffice sowie phasenweise geschlossener Gastronomiebetriebe und Kultureinrichtungen stark reduziert war und hygienebedingt plattformvermittelte Mobilitätsdienstleistungen kaum nachgefragt waren - also in einer Phase ungeplanter Alternativen zur motorisierten Massenmobilität und gleichzeitig einer Phase verstärkter Wahrnehmung von vergeschlechtlichter Arbeitsteilung und unbezahlter häuslicher Sorgearbeit. Wir sehen zwar wie viele andere kritische Stadtforscher_innen die Ermöglichung von alternativen Strukturen in Krisenzeiten, es ist jedoch keine Abkehr von einer autogerechten Stadt zu erkennen. Wir halten daher unsere Argumentation für europäische Städte auch in der Post-Pandemie-Phase für relevant.

[3] Vgl. das Forschungsprojekt „Geschlechtergerechte Stadtentwicklung in Smart City Kontexten“ im Elisabeth-List-Fellowship-Programm der Universität Graz (2020/21) von Anke Strüver, Sybille Bauriedl, Henk Wiechers, Yannick Ecker und Marcella Rowek (https://fellowship-geschlechterforschung.uni-graz.at/de/projekte/smart-city).

[4] Intermediäre Plattformen vermitteln digitale Inhalte und stellen keine eigenen Produkte oder Dienstleistungen bereit. Darunter fallen Suchmaschinen (z. B. Google, Yahoo), soziale Netzwerke (z. B. Facebook), App-Plattformen (z. B. Apple App Store), User-generatedcontent-Plattformen (z. B. Instagram) oder Verkaufsplattformen (z. B. eBay).

[5] Dies gilt auch für Nicht-Weiße, Transgender und Menschen, die sich mit nicht-normativen Sexualitäten identifizieren und sich vor Aggressionserfahrungen im öffentlichen Raum schützen.

\section{Autor_innen}

Sybille Bauriedl ist Geographin mit den Arbeitsschwerpunkten Klimagerechtigkeit, digitale Transformation, Energiewende, Politische Ökologie, feministische Geographie und postkoloniale Geographie.

sybille.bauriedl@uni-flensburg.de

Henk Wiechers ist Stadtgeograph. Seine Arbeitsschwerpunkte sind Wohnungslosigkeit, Digitalisierung und qualitative Forschungsmethoden.

henk.wiechers@uni-flensburg.de

\section{Literatur}

Alonso-Almeida, María del Mar (2019): Carsharing. Another gender issue? Drivers of carsharing usage among women and relationship to perceived value. In: Travel Behaviour and Society 17, 36-45.

Balkmar, Dag / Mellström, Ulf (2018): Masculinity and autonomous vehicles. A degendered and resegregated future system of automobility. In: Transfers. Interdisciplinary Journal of Mobility Studies 8/1, 44-63.

Barns, Sarah (2019): Negotiating the platform pivot. From participatory digital ecosystems to infrastructures of everyday life. In: Geography Compass 13/9, 1-13. 
Barns, Sarah (2020): Platform urbanism. Negotiating platform ecosystems in connected cities. Singapore: Springer Nature.

Bauriedl, Sybille (2020): Luxusmodelle für den Klimaschutz? E-Carsharing in Großstädten als Experimentierfeld der Automobil- und Digitalwirtschaft. In: Achim Brunnengräber / Tobias Haas (Hg.): Baustelle Elektromobilität. Sozialwissenschaftliche Perspektiven auf die Transformation der (Auto-)Mobilität. Bielefeld: transcript, 161-180.

Bauriedl, Sybille / Strüver, Anke (Hg.) (2018): Smart City. Kritische Perspektiven auf die Digitalisierung in Städten. Bielefeld: transcript.

Berliner Verkehrsbetriebe (2020): Die Jelbi-Mobilitätspartner. https://www.jelbi.de/ mobilitaetspartner (letzter Zugriff am 14.5.2020).

Bosler, Micha / Jud, Christopher / Herzwurm, Georg (2017): Connected-car-services. Eine Klassifikation der Plattformen für das vernetzte Automobil. In: HMD Praxis der Wirschaftsinformatik 54/6, 1005-1020.

Church, Andrew / Frost, Martin / Sullivan, Katie (2000): Transport and social exclusion in London. In: Transport Policy 7, 195-205.

Daum, Timo (2018): Das Auto im digitalen Kapitalismus. Berlin: Rosa-Luxemburg-Stiftung.

Docherty, Iain / Marsden, Greg / Anable, Jillian (2018): The governance of smart mobility. In: Transportation Research Part A 115, 114-125.

Giesel, Flemming / Nobis, Claudia (2016): The impact of carsharing on car ownership in German cities. In: Transportation Research Procedia 19 (C), 215-224.

Graham, Mark (2020): Regulate, replicate, and resist. The conjunctural geographies of platform urbanism. In: Urban Geography 41/3, 453-457.

Graham, Stephen (2002): Bridging urban digital divides? Urban polarisation and information and communications technologies (ICTs). In: Urban Studies 39/1, 33-56.

Green Mobility A/S (2020): Our Journey. https://partner.greenmobility.com/our-journey (letzter Zugriff am 14.5.2020).

Groth, Sören (2019a): Multioptionalität: Ein neuer („alter“) Terminus in der Alltagsmobilität der modernen Gesellschaft? In: Raumforschung und Raumordnung 77/1, 17-34.

Groth, Sören (2019b): Multimodal divide. Reproduction of transport poverty in smart mobility trends. In: Transportation Research Part A: Policy and Practice 125, 56-71.

Groth, Sören (2019c): Von der automobilen zur multimodalen Gesellschaft? Multioptionalität als Voraussetzung für multimodales Verhalten. Bielefeld: transcript.

Groupe PSA (2017): Groupe's Websites. https://www.groupe-psa.com/en/groups-website (letzter Zugriff am 14.5.2020).

Hanson, Susan (2010): Gender and mobility. New approaches for informing sustainability. In: Gender, Place and Culture 17/1: 5-23.

Heeg, Susanne / Rosol, Marit (2007): Neoliberale Stadtpolitik im globalen Kontext. Ein Überblick. In: Prokla. Zeitschrift für kritische Sozialwissenschaft 37/149, 491-510.

Hildebrand, Julia M. / Sheller, Mimi (2018): Media ecologies of autonomous automobility. Gendered and racial dimensions of future concept cars. In: Transfers. Interdisciplinary Journal of Mobility Studies 8/1, 64-85.

Kent, Jennifer L. / Dowling, Robyn (2013): Puncturing automobility? Carsharing practices. In: Journal of Transport Geography 32, 86-92.

Kenyon, Susan / Lyons, Glenn / Rafferty, Jackie (2002): Transport and social exclusion. Investigating the possibility of promoting inclusion through virtual mobility. In: Journal of Transport Geography 10/3, 207-219.

Kitchin, Rob (2018): Towards a genuinely humanizing smart urbanism. The programmable city working paper 42. https://osf.io/preprints/socarxiv/5jkx4 (letzter Zugriff am 12.5.2020).

Law, Robin (1999): Beyond „women and transport“. Towards new geographies of gender and daily mobility. In: Progress in Human Geography 23/4, 567-588.

Lee, Ashlin / Mackenzie, Adrian / Smith, Gavin J. D. / Box, Paul (2020): Mapping platform urbanism. Charting the nuance of the platform pivot. In: Urban Planning 5/1, 116-128.

Liyanage, Sohani / Dia, Hussein / Abduljabbar, Rusul / Bagloee, Saeed (2019): Flexible mobility on-demand. An environmental scan. In: Sustainability 11/5, 1-39.

Lucas, Karen (2012): Transport and social exclusion. Where are we now? In: Transport Policy 20, 105-113.

MaaS Global Oy (2020): Find your plan. https://whimapp.com/plans (letzter Zugriff am 14.5.2020). 
Marvin, Simon / Luque-Ayala, Andrés / McFarlane, Colin (Hg.) (2016): Smart urbanism. Utopian vision or false dawn? New York: Routledge.

Mobility Genossenschaft (2020): Die Mobility Genossenschaft. https://www.mobility.ch/ de/mobility-genossenschaft/ueber-uns (letzter Zugriff am 14.5.2020).

Motsiefer, Hendrik (2019): Warum BMW und Daimler ihr Carsharing-Angebot zurückfahren. In: Der Tagesspiegel, 19.12.2019. https://www.tagesspiegel.de/wirtschaft/ extrem-schwierige-realitaeten-warum-bmw-und-daimler-ihr-carsharing-angebotzurueckfahren/25351634.html (letzter Zugriff am 14.5.2020).

Pangbourne, Kate / Mladenović, Miloš N. / Stead, Dominic / Milakis, Dimitris (2020): Questioning mobility as a service. Unanticipated implications for society and governance. In: Transportation Research Part A: Policy and Practice 131, 35-49.

Rid, Wolfgang / Parzinger, Gerhard / Grausam, Michael / Müller, Ulrich / Herdtle, Carolin (2018): Carsharing in Deutschland. Potenziale und Herausforderungen, Geschäftsmodelle und Elektromobilität. Wiesbaden: Springer.

Sadowski, Jathan (2020): Who owns the future city? Phases of technological urbanism and shifts of sovereignity. In: Urban Studies, 1-13.https://doi.org/10.1177/0042098020913427

Shaheen, Susan / Martin, Elliot / Totte, Hannah (2020): Zero-emission vehicle exposure within U.S. carsharing fleets and impacts on sentiment toward electric-drive vehicles. In: Transport Policy 85, 23-32.

ShareNow GmbH (2020): Über ShareNow. https://www.share-now.com/de/de/faq/aboutshare-now/ (letzter Zugriff am 14.5.2020).

Sheller, Mimi (2015): Racialized mobility transitions in Philadelphia. Connecting urban sustainability and transport justice. In: City \& Society 27/1, 70-91.

Sheller, Mimi / Urry, John (2006): The new mobilities paradigm. In: Environment and Planning A: Economy and Space 38/2, 207-226.

Singh, Yamini J. (2019): Is smart mobility also gender-smart? In: Journal of Gender Studies 29/7, 1-15.

Söderström, Ola / Paasche, Till / Klauser, Francisco (2014): Smart cities as corporate storytelling. In: City 18/3, 307-320.

Srnicek, Nick (2016): Platform capitalism. Cambridge, Malden: Polity Press.

Stehlin, John / Hodson, Michael / McMeekin, Andrew (2020): Platform mobilities and the production of urban space. Toward a typology of platformization trajectories. In: Environment and Planning A: Economy and Space, 1-19.

Strüver, Anke / Bauriedl, Sybille (2020): Smart Cities und sozialräumliche Gerechtigkeit. Wohnen und Mobilität in Großstädten. In: Christine Hannemann / Frank Othengrafen / Jörg Pohlan / Brigitta Schmidt-Lauber / Rainer Wehrhahn / Simon Güntner (Hg.), Jahrbuch StadtRegion 2019/2020. Schwerpunkt: Digitale Transformation. Wiesbaden: Springer, 91-112.

Stüber, Jürgen (2019): „Wir lösen den Unterschied zwischen Autovermietung und Carsharing auf". https://www.gruenderszene.de/automotive-mobility/sixt-carsharinginterview-gabriel (letzter Zugriff am 14.5.2020).

Uteng, Tanu Priya (2011): Gendered bargains of daily mobility. Citing cases from both urban and rural settings. The World Bank.

Uteng, Tanu Priya / Christensen, Hilda Rømer / Levin, Lena (Hg.) (2019): Gendering smart mobilities. London: Routledge.

Uteng, Tanu Priya / Cresswell, Tim (Hg.) (2008): Gendered mobilities. Towards a holistic understanding. London: Routledge.

van Dijck, José / Poell, Thomas / De Waal, Martijn (2018): The platform society. Public values in a connective world. Oxford: Oxford University Press.

Velaga, Nagendra R. / Beecroft, Mark / Nelson, John D. / Corsar, David / Edwards, Peter (2012): Transport poverty meets the digital divide. Accessibility and connectivity in rural communities. In: Journal of Transport Geography 21, 102-112.

Volkswagen AG (2020): WeShare expandiert ins europäische Ausland. https://www.volkswagenag.com/de/news/2020/o1/WeShare.html (letzter Zugriff am 14.05.2020).

Volvo Car Mobility (2020): Stations. Find cars in your neighbourhood. https://m.co/se/ en-US/stations (letzter Zugriff am 14.5.2020).

WBGU - Wissenschaftlicher Beirat der Bundesregierung Globale Umweltveränderungen (2019): Hauptgutachten. Unsere gemeinsame digitale Zukunft. www.wbgu.de/de/ publikationen/publikation/unsere-gemeinsame-digitale-zukunft (letzter Zugriff am 12.5.2020). 


\section{Platform urbanism in Europe. Digital divide and gendered Smart Mobility}

Smart Cities are associated with great promises of improved urban quality of life through digital technologies. This technology-optimistic urban development follows on from the modernization promises of the Entrepreneurial City. A wide variety of supply tasks in cities are now being provided via online platforms operated by international IT companies and supported by digital infrastructures in public spaces. In urban studies, this process is termed platform urbanism. 